\title{
Extending Horizon of Robotic Surgery to Bladder-Preserving Approach for Vesical Paraganglioma: Rare Case with Unusual Presentation
}

\author{
R. Ranjan, MCh (resident), A. Mittal, MCh, V. Panwar, MCh, T.A. Narain, MCh, \\ H.S. Talwar, MCh (resident), and K.J. Mammen, MCh
}

\begin{abstract}
Background: Vesical paraganglioma is rare and accounts for $<0.1 \%$ of all urinary bladder tumors. They are mostly functional because of secretion of catecholamines and clinical presentation may mimic like a hyperfunctioning adrenal pheochromocytoma. They are easily misdiagnosed as urothelial malignancy and adequate perioperative attention is not provided.

Case presentation: We hereby report a case of 55-year-old Indian lady with silent vesical paraganglioma at anatomically difficult location of bladder neck managed with robot-assisted excision of mass and bladder preservation.

Conclusion: Surgery is the mainstay of the treatment that requires total excision of mass. However, minimally invasive bladder-preserving approach should be always kept as an option, if feasible. Robot assistance can help in bladder preservation even in difficult anatomic locations.
\end{abstract}

Keywords: vesical paraganglioma, bladder tumor, bladder preservation, robotic surgery

\section{Introduction}

$\mathbf{P}$ ARAGANGLIOMA OF URINARY bladder is extremely rare tumor of chromaffin tissue that takes origin from sympathetic innervation. ${ }^{1}$ These are usually functional, but their silent presentation is quite dangerous as they are easily misdiagnosed and adequate perioperative attention is not provided. ${ }^{1,2}$ Our index case presented as silent paraganglioma, which was managed by robot-assisted bladder-preserving approach.

\section{Case Report}

A 55-year-old Indian lady with no comorbidity presented with mild discomfort at lower abdomen for 6 years. MRI pelvis showed well-circumscribed lobulated submucosal round-shaped mass measuring $4 \times 4 \times 3 \mathrm{~cm}$ arising from bladder base anterior to the urethral opening. It was mild hyperintense signal intensity on $\mathrm{T} 1$ image and heterogeneously hyperintense on $\mathrm{T} 2$ image with peripheral $\mathrm{T} 2$ hypointense rim suggesting capsule. There was restricted diffusion with low apparent diffusion coefficient value on diffusion weighted imaging. Postcontrast images revealed avid fairly homogenous enhancement and multiple arterial feeders arising from bilateral internal iliac artery. All these features were suggestive of paraganglioma (Fig. 1). Cystoscopy was done under local anesthesia and revealed $3 \times 2 \mathrm{~cm}$ bulge at an- terior wall with overlying normal mucosa (Fig. 2). Serum metanephrine $(860 \mathrm{pg} / \mathrm{mL})$ and normetanephrine $(966 \mathrm{pg} / \mathrm{mL})$ was raised significantly. 123I-Meta-iodobenzylguanidine (MIBG) scan revealed increased uptake limited to lesion at bladder neck (Fig. 3).

Bladder preservation surgery was planned after adequate sympathetic blockage and hydration. Robot-assisted excision of vesical paraganglioma was done (Fig. 4). It was done in Trendelenburg position with one supraumbilical camera port $(8 \mathrm{~mm})$, two left-sided port $(8 \mathrm{~mm})$, one right-side port $(8 \mathrm{~mm})$, and one right-side assistant port $(12 \mathrm{~mm})$. Transperitoneally we reached till bladder neck through fine dissection and tumor was identified by its bulge over the anterior surface on the bladder just proximal to the neck in fully distended bladder. Tumor was excised with margin of $5 \mathrm{~mm}$ along with bladder mucosa. Defect was closed in two layers and Foley catheter was kept for 10 days. Intra- and postoperative period was uneventful. Histopathology report revealed well-differentiated tumor, synaptophysin and chromogranin positive, suggestive of paraganglioma.

\section{Discussion}

Paragangliomas are accumulations of chromaffin tissue derivative of primitive cells of the neural crest. ${ }^{2}$ Starting from 28th week of embryologic development, the paraganglionic

Department of Urology, All India Institute of Medical Science (AIIMS), Rishikesh, Uttarakhand, India. 
FIG. 1. MRI showing welldefined enhancing lobulated mass in submucosa near bladder neck. (A) Axial and (B) sagittal view.
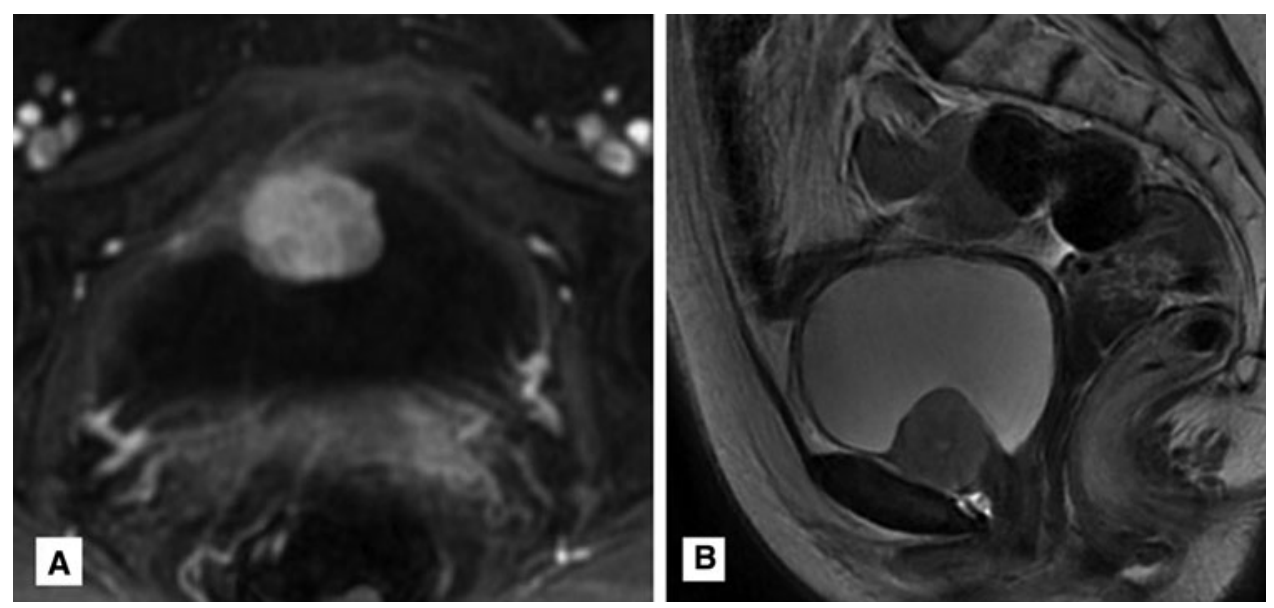

system undergoes progressive regression. ${ }^{2}$ Aside from adrenal medulla, only minor remnants and organ of Zuckerkandl remain in adult. There might be persistence of paraganglia around the vesical wall and the ureter. Paraganglioma arising from the clusters of residual cells are usually functional and symptomatic. ${ }^{1,2}$ These are usually benign, but $15 \%-20 \%$ tumors may show malignant behavior that cannot be differentiated on the basis of histology report. ${ }^{3}$ Malignancy can be confirmed only after either local invasion or distant metastases. ${ }^{3}$

Functional paragangliomas may give rise to clinical presentation similar to the hyperfunctioning adrenal pheochromocytoma. $^{3}$ Secretion of catecholamine occurs with increased bladder pressure when it contracts and consequently triggers sympathomimetic attack. ${ }^{2}$ It is typically recognized by hypertensive crisis and syncope, provoked by micturition, defecation, ejaculation, sexual activities, or vesical instrumentation contrary to silent presentation as depicted in our index case. ${ }^{3}$

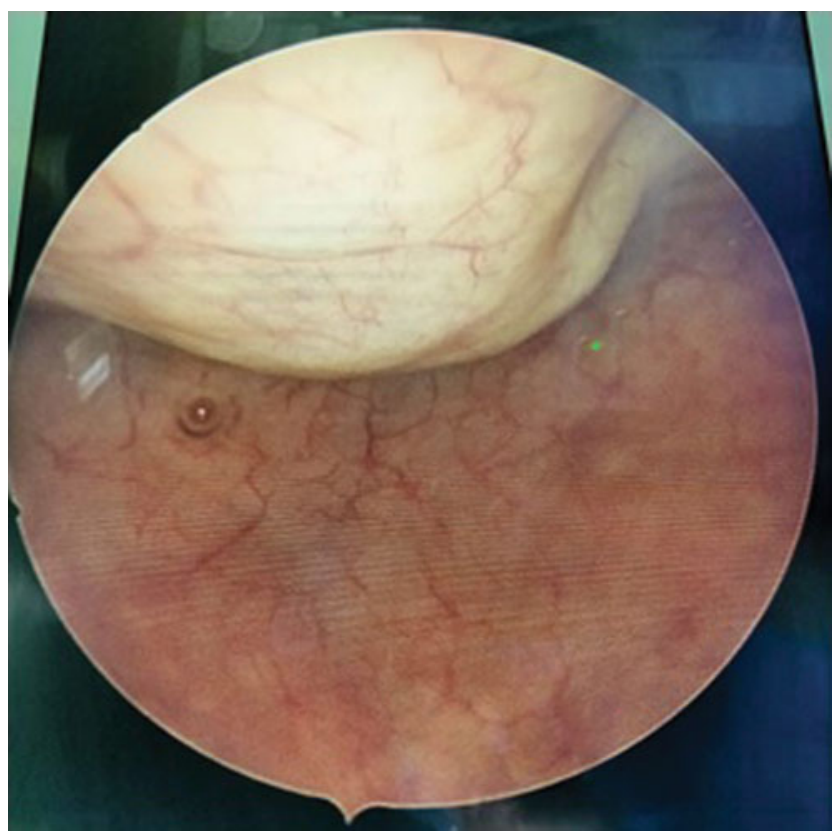

FIG. 2. Cystoscopy showing bulge at anterior wall with overlying normal mucosa.
Ultrasound is the first diagnostic imaging modality, which reveals highly vascular nature of this tumor. ${ }^{1,2} \mathrm{CT}$ or MRI is useful for localization of paraganglioma and can detect majority of tumors of size $>1 \mathrm{~cm}$ and locate multifocal tumors and also metastases of malignant tumors. ${ }^{1,3}$ Sensitivity of CT is $82 \%$, but MRI is better than CT for the diagnosis of vesical paraganglioma. ${ }^{1,2} \mathrm{~T} 1$ sequence images allow optimum assessment of perivesical extension and detection of pelvic lymphadenopathy. ${ }^{2} \mathrm{~T} 2$ sequence images are required to assess the infiltration of bladder wall, which has characteristic high signal intensity. ${ }^{2}$ Scintigraphy scan with ${ }^{131}$ I-MIBG is important by virtue of its high affinity and storage in the chromaffin cells, ensuing high sensitivity as well as specificity and also opportunity for the scanning of whole body. ${ }^{1,3}$ Plasma or urinary catecholamines (metanephrine and normetanephrine) are done to see the function of the paraganglioma. ${ }^{3}$ Surgical excision of the mass is mainstay of treatment. ${ }^{1-3}$ Bladder preservation should be attempted but in situations such as large mass or difficult anatomical location cystectomy is done. Transurethral resection of bladder tumor (TURBT) is usually inadequate and not advisable because catecholamine crises can be triggered during the resection. ${ }^{1,2}$ However, TURBT can be an option for hormonally inactive paragangliomas that are small and easily accessible transurethrally for complete removal. ${ }^{4}$ Recurrence rate is high, so life-long follow-up with appropriate history, annual plasma catecholamines level measurement, and cystoscopy is essential. ${ }^{1,2}$

In our case, we did robot-assisted laparoscopic excision of vesical paraganglioma by bladder-preserving approach. With robotic assistance surgery in the deep pelvis was much easier because of better ergonomic vision and fine movements. Intra- and postoperative period was uneventful. No drain was placed and patient was discharged on postoperative day 2 . After 6 months of follow-up patient did not have any recurrence and no abnormal uptake on MIBG scan.

\section{Conclusion}

Vesical paraganglioma is a rare tumor and may radiologically, cystoscopically, and histologically mimic a urothelial neoplasm. It should be considered in the differential diagnosis of urinary bladder tumors, especially in patients without the specific symptoms. Surgery is the mainstay of the treatment that requires total excision of mass. However, 

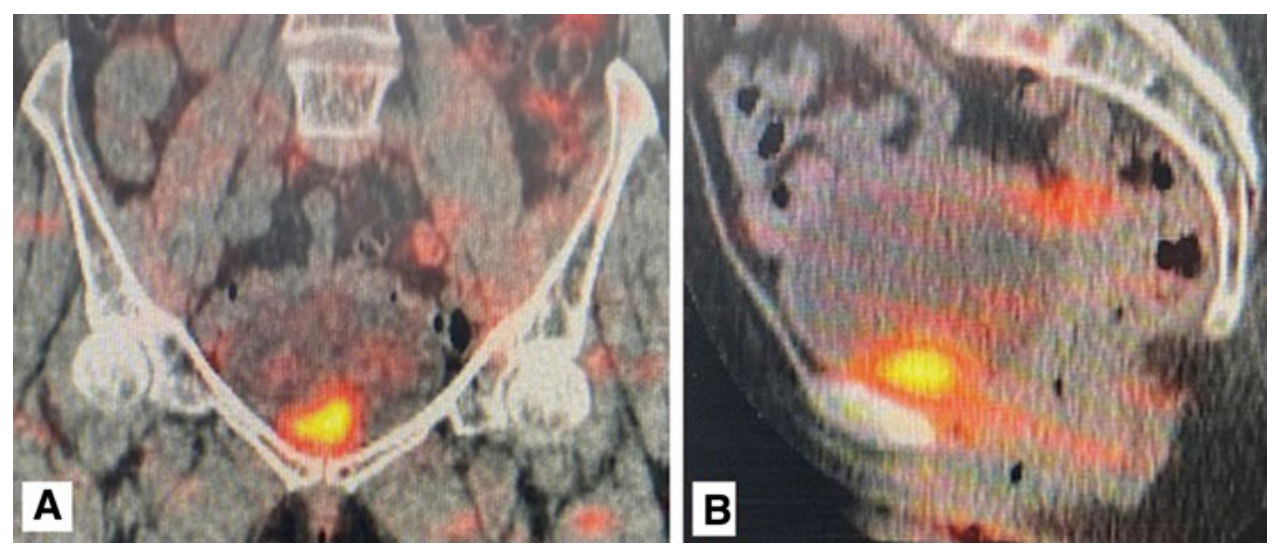

FIG. 3. 123I-meta-iodobenzylguanidine scan showing increase uptake at bladder neck only. (A) Coronal and (B) sagittal view.
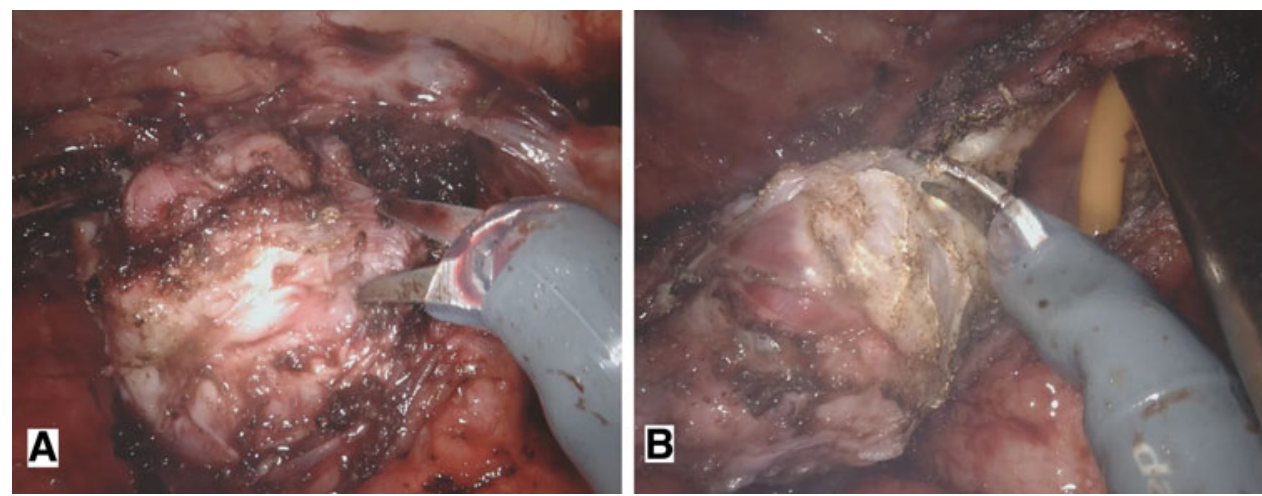

FIG. 4. Intraoperative photographs (A, B).

minimally invasive bladder-preserving approach should be always kept as an option, if feasible. Robot assistance can help in bladder preservation even in difficult anatomic locations. Life-long follow-up is necessary because of its high recurrence rate.

\section{Disclosure Statement}

No competing financial interests exist.

\section{Funding Information}

No funding was received for this article.

\section{References}

1. Hermi A, Ichaoui H, Kacem A, et al. Functional bladder paraganglioma treated by partial cystectomy. Case Rep Urol 2019;2019:1-4.

2. Pastor-Guzmán JM, López-García S, Giménez-Bachs JM, et al. Paraganglioma of the bladder: Controversy regarding treatment. Urol Int 2004;73:270-275.

3. Ranaweera M. Bladder paraganglioma: A report of case series and critical review of current literature. World J Clin Cases 2014;2:591.

4. Zhang B, Fu Z, Liu L, Qiao B, Liu C. Non-functional paraganglioma of urinary bladder managed by transurethral resection. Int Braz J Urol 2019;45:910-915.

\author{
Address correspondence to: \\ A. Mittal, MCh \\ Department of Urology \\ All India Institute of Medical Science (AIIMS) \\ Rishikesh 249202 \\ Uttarakhand \\ India
}

E-mail: drmittal.ankur@gmail.com

Cite this article as: Ranjan R, Mittal A, Panwar V, Narain TA, Talwar HS, Mammen KJ (2020) Extending horizon of robotic surgery to bladder-preserving approach for vesical paraganglioma: rare case with unusual presentation, Journal of Endourology Case Reports 6:4, 319-321, DOI: 10.1089/cren.2020.0077. 